\title{
ÉVOLUTION DU NOMBRE D'ACCÈS DIRECTS COMMERCIAUX À TRANSPAC (1979 - 1985)
}

\author{
Valérie Schafer \\ Métropolis | Flux
}

$2005 / 4-n^{\circ} 62$

pages 75 à 80

ISSN 1154-2721

Article disponible en ligne à l'adresse:

http://www.cairn.info/revue-flux-2005-4-page-75.htm

Pour citer cet article :

Schafer Valérie, « Évolution du nombre d'accès directs commerciaux à Transpac (1979 - 1985) »,

Flux, 2005/4 n62, p. 75-80.

Distribution électronique Cairn.info pour Métropolis.

(c) Métropolis. Tous droits réservés pour tous pays.

La reproduction ou représentation de cet article, notamment par photocopie, n'est autorisée que dans les limites des conditions générales d'utilisation du site ou, le cas échéant, des conditions générales de la licence souscrite par votre établissement. Toute autre reproduction ou représentation, en tout ou partie, sous quelque forme et de quelque manière que ce soit, est interdite sauf accord préalable et écrit de l'éditeur, en dehors des cas prévus par la législation en vigueur en France. II est précisé que son stockage dans une base de données est également interdit. 


\section{HISTOIRE DE COURBE}

\section{Évolution du nombre d'accès directs commerciaux à Transpac (1979 - 1985)}

Valérie Schafer

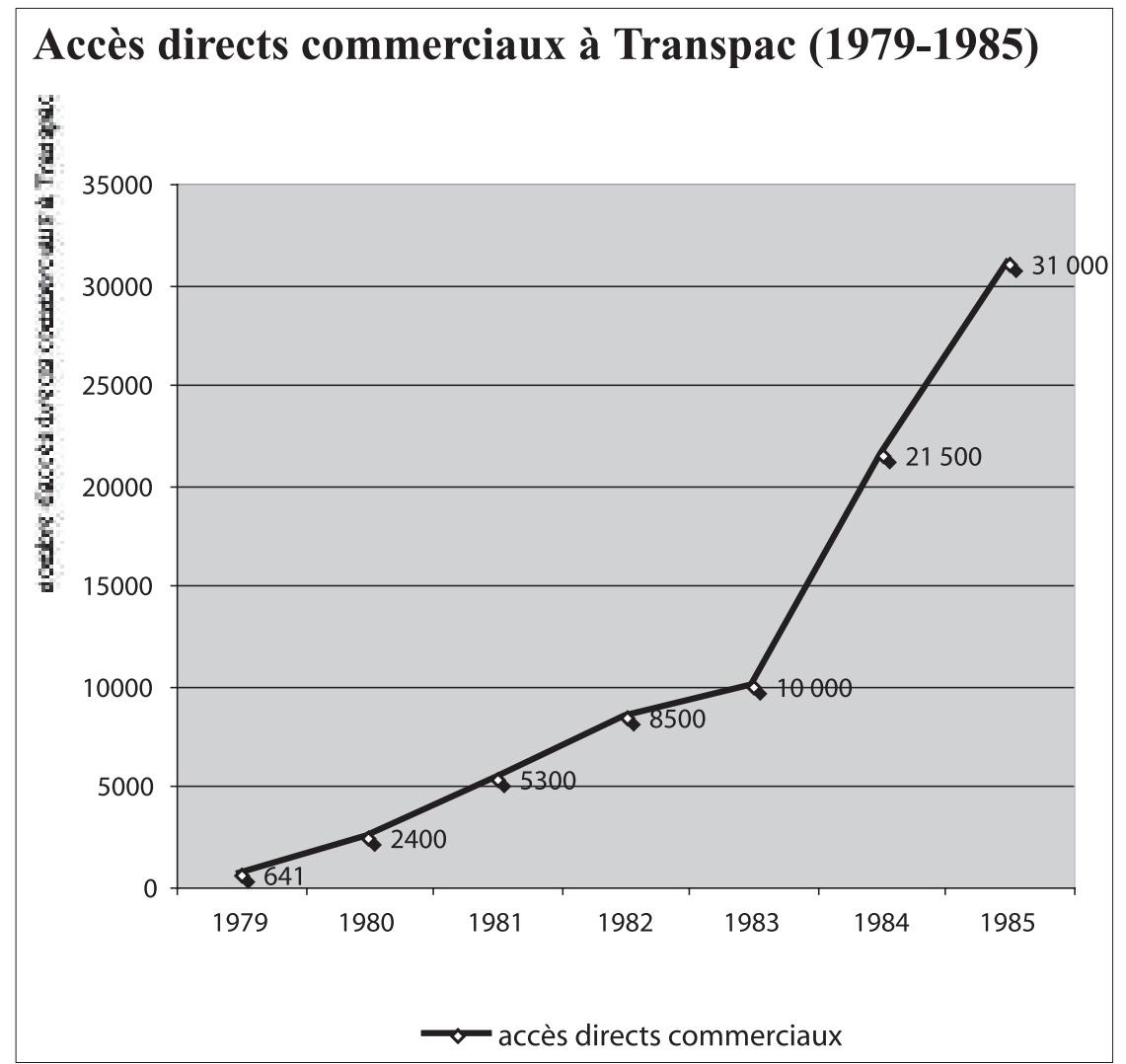

Lors de son ouverture en 1978, le réseau public de données Transpac se veut pour les télécommunicants (la Direction Générale des Télécommunications ou DGT et le Centre National d'Études des Télécommunications ou CNET) une réponse à plusieurs préoccupations :

- la multiplication des liaisons spécialisées
- la satisfaction des besoins des PME

- le développement d'un réseau utilisant une nouvelle technologie qui met en concurrence les télécommunicants et les informaticiens de I'IRIA (Institut de Recherches en Informatique et Automatique), et est destinée à un avenir prometteur, notamment dans le protocole
TCP/IP: la commutation de paquets.

Ouvert en 1978, ce réseau va connaître une croissance rapide, d'abord tournée vers la satisfaction des besoins des entreprises, puis supporter le trafic du minitel, et placer la France parmi les précurseurs dans le champ des réseaux publics et dans celui de la commutation par paquets.

Pour celui qui s'intéresse aux transmissions de données, le réseau Transpac est une étape obligée. À partir de données collectées dans des publications d'acteurs du réseau Transpac (notamment les données proposées par Guy Pichon dans un article présenté lors du $7^{\mathrm{e}}$ colloque sur l'histoire de l'informatique (1)), d'archives et de périodiques dédiés aux télécommunications, telle la Revue Française des Télécommunications, nous avons donc choisi d'interroger une courbe concernant le nombre d'accès directs commerciaux à Transpac, afin d'évaluer le succès de ce réseau.

\section{Quelques éléments de définition}

C'est en constatant que Guy Pichon, ancien acteur de Transpac (2), ou les collaborateurs de F. Du Castel dans un ouvrage diffusé en interne par France Telecom (3), utilisent le nombre d'accès directs à Transpac, qu'est venue l'idée d'analyser ces chiffres. Aussi les données proposées pour cette courbe sont celles fournies dans son article par G. Pichon (4). Elles sont parfois approximatives (" Le cap des 21500 accès directs commerciaux est franchi en fin d'année » (5)) et non four- 
nies pour l'année 1981 (le nombre des accès directs en 1981 provient d'un bilan d'activités (6)).

Si ce critère des accès directs est retenu par des acteurs du monde des télécommunications, est-ce parce qu'il offre une vision pertinente de l'évolution de Transpac?

Il convient tout d'abord de définir ce qu'est un accès direct. Dès son origine, Transpac offre en effet plusieurs moyens d'accès au réseau. II faut distinguer:

- l'accès direct (par liaisons spécialisées)

Dans ce cas l'équipement informatique est relié directement à l'un des points d'accès du réseau. II est abonné au réseau.

Un centre de traitement abonné gérant un grand nombre de terminaux à travers Transpac peut avoir à échanger un trafic important avec le réseau. C'est alors un raccordement multiligne qui est utilisé, constitué d'un faisceau de lignes reliant le centre de traitement à un commutateur.

- l'accès par le réseau téléphonique commuté ou télex

Deuxième moyen d'accès au réseau Transpac à ses débuts, ce mode consiste pour l'abonné du réseau téléphonique ou télex à communiquer avec un abonné Transpac en appelant sur son réseau un des deux types d'accès suivants: une entrée banalisée (par un système de numérotation), ou une entrée réservée. Ce cas n'est pas pris en compte par la courbe, de même que n'est pas prise en compte non plus la possibilité ensuite d'accéder à Transpac par les Points d'accès vidéotex (PAV), mode d'accès privilégié pour le grand public utilisant le minitel et les services télétel. Le raccordement au réseau Transpac permet ainsi d'ouvrir, en 1984, la taxation "kiosque », système qui assure une partie du succès du minitel en multipliant l'offre de services, tout en assurant une rémunération simple des serveurs.

La courbe ne prend donc en compte que le premier moyen d'accès à Transpac, l'accès direct, et plus précisément les accès directs commerciaux (les accès commerciaux sont relatifs à des clients réels contrairement aux accès de service, utilisés pour la gestion du réseau, le raccordement des terminaux d'exploitation, la gestion des liaisons inter-commutateurs). Elle a pour date d'origine l'année 1979, première année de service du réseau (ouvert fin 1978) et s'achève en 1985, une année essentielle dans l'histoire de Transpac. En effet, alors que le réseau, victime de son succès et de celui du minitel, connaît des difficultés techniques et une dégradation des performances qui impliquent des corrections au cours de cette année (7), les premiers commutateurs de deuxième génération sont introduits dans le réseau, une filiale INTERPAC est créée pour le domaine international, le chiffre d'affaires de la société atteint le milliard de francs. Au-delà, l'histoire de Transpac, sans se confondre avec celle du minitel, connaît une évolution que la courbe des accès directs ne permet plus de suivre de façon entièrement satisfaisante, alors qu'en 1989 par exemple, la Cour des Comptes note dans un rapport (8) que le réseau Transpac doit la moitié de son trafic au minitel.
En amont de cette courbe: la décision de lancer un réseau public de données à commutation de paquets

Le choix d'analyser les raccordements directs à Transpac permet de partir de données concernant les usagers auxquels, à son origine, le réseau est destiné: le monde professionnel. En effet, à ses débuts, Transpac est tourné vers les entreprises, notamment les PME, et cherche à recruter dans cette sphère ses abonnés.

Dès les années 1970, le CNET étudiait la possibilité de créer un réseau public de données utilisant la commutation électronique.

Un tournant a lieu en 1973 lorsque la DGT, en la personne de son Directeur Général L. J. Libois, annonce le lancement des études devant déboucher sur la création d'un réseau public à commutation de paquets avant fin 1975.

La DGT répond à une double pression: d'une part le projet Cyclades des informaticiens de I'IRIA est en train de monter en puissance et s'appuie sur la technique de la commutation de paquets (9) qu'expérimente aussi le CNET (la première démonstration officielle de Cyclades a lieu en novembre 1973 en présence des Ministres de l'Industrie et des PTT, ce qui est délicat pour les télécommunicants). D'autre part, les grands utilisateurs, regroupés dans un groupement spécialisé animé par Pierre Lhermitte, le GERCIP, qui réunit entre autres la Société Générale, EDF, ou Saint-Gobain, se demandent s'ils ne vont pas essayer de mettre en place un réseau partagé de données, 
basé sur la commutation de messages ou de paquets. Ils étudient la possibilité de louer des lignes aux PTT et de faire eux-mêmes leur propre réseau (10), et inquiètent certains télécommunicants qui craignent que cela porte atteinte à leur rôle et au monopole.

En 1975 est lancé l'appel d'offre Transpac, et sept consortiums y répondent. C'est la SESA qui emporte le marché. Le réseau Transpac est ouvert en décembre 1978, après la création en 1977 d'une société dont $77 \%$ sont détenus par l'État. Transpac est un des premiers réseaux publics de transmission de données au monde et s'impose comme le réseau à commutation de paquets français, desservant dès ses débuts tout le territoire national (11). La société Transpac (décision interministérielle de 1974), société anonyme de droit privé, est rapidement bénéficiaire et le réseau va ensuite supporter le développement du trafic minitel, décision prise après quelques polémiques au sein des télécommunicants, notamment à cause du statut de la société Transpac que certains ont pu considérer comme un outil du « démantèlement $d u$ service public » (12).

\section{Transpac: une réussite technique et commerciale, pour un réseau " au service des entreprises "}

La courbe fait apparaitre une croissance rapide et spectaculaire $d u$ nombre des accès directs commerciaux à Transpac. Entre 1979 et 1985 ils ont été multipliés par 48 environ. Dans ses premières années, Transpac a vu les raccordements directs plus que tripler
(3,74 fois plus de raccordements en 1980 qu'en 1979, contre environ 1,45 fois plus de raccordements entre 1984 et 1985 - mais en valeur absolue 9500 raccordements directs supplémentaires au cours de cette année 1985 contre seulement 1760 entre 1979 et 1980). Le chiffre d'affaires de la société suit en parallèle une évolution croissante (150 millions de francs en 1982, 500 millions de francs en 1984, 1 milliard en 1985, mais l'évolution des tarifs limite la comparaison des deux courbes).

Surtout, Transpac en 1985 dépasse les objectifs quantitatifs initialement définis par les télécommunicants. À l'ouverture du réseau en 1979, les télécommunicants espéraient pour 1985 environ 25000 abonnés (13) (ils sont 31500 fin 1985), et jusqu'en 1985 un taux de croissance moyen de 25\% (14), lui aussi largement dépassé.

Les objectifs de Transpac à son ouverture répondent, comme nous l'avons vu précédemment, à la concurrence des informaticiens dans le domaine des réseaux, à la pression des " gros clients » en matière de transmissions de données mais aussi au souci des télécommunicants de s'adapter à la croissance rapide de la demande en téléinformatique. Non seulement ils s'inquiètent du recours des entreprises aux liaisons spécialisées (15), qui échappent partiellement aux Télécommunications même si ce sont elles qui les louent, mais ils cherchent à être abordables par les moyens et les petits utilisateurs, et à favoriser la décentralisation en desservant tout le territoire et en supprimant l'incidence de la distance sur les coûts (16). Transpac est en effet accessible en tout point du territoire français et présente l'avantage d'offrir une facturation indépendante de la distance. Outre le montant de l'abonnement lié à la vitesse de transmission retenue par le client, l'utilisation du service est principalement taxée selon le volume d'informations transmis et dans une très faible mesure en fonction de la durée. Ainsi les petits utilisateurs se retrouvent dans une situation d'égalité par rapport aux plus gros, de même que les utilisateurs très décentralisés n'ont rien à envier à ceux qui sont plus proches du centre de traitement. Or, comme le note la Revue des télécommunications, n55, d'avril 1985, les PME et les professions libérales, en raison de l'importance de l'investissement, n'accédaient jusqu'à la création de Transpac qu'exceptionnellement à l'univers de l'informatique, alors qu'en 1978 le rapport Nora-Minc sur l'informatisation de la société (17) préconise de faciliter le raccordement des petits et moyens utilisateurs.

Aussi l'analyse de cette courbe impliquerait de la mettre en parallèle avec celle des liaisons spécialisées, mais aussi d'établir des nuances entre les utilisateurs, dont la présentation est ici indifférenciée, afin de voir quels sont les usagers de Transpac.

L'analyse au 31 décembre 1984 des utilisateurs de Transpac par secteur d'activités (tableau page suivante) montre par l'exemple l'accès des professions libérales au service Transpac. S'il est certes encore modeste, il témoigne de l'ouverture vers des « petits clients " à côté des administrations ou des assurances, qui sont parmi les utilisateurs initiaux des transmissions 


\begin{tabular}{|c|c|c|}
\hline $\begin{array}{l}\text { Utilisateurs de Transpac } \\
\text { par secteur d'activités }\end{array}$ & Usagers (\%) & Nombre de lignes (\%) \\
\hline Administrations & 13,2 & 21,9 \\
\hline Entreprises nationalisées & 4,5 & 6,2 \\
\hline Banque et finance & 12,7 & 19,2 \\
\hline Assurances & 17,6 & 8,9 \\
\hline Industrie & 21,6 & 17,7 \\
\hline Commerce & 9,8 & 8,8 \\
\hline Services & 7,4 & 5,7 \\
\hline $\begin{array}{l}\text { Constructeurs } \\
\text { «informatiques» }\end{array}$ & 3,4 & 3,4 \\
\hline $\begin{array}{l}\text { Sociétés de service } \\
\text { informatique }\end{array}$ & 7,6 & 7,7 \\
\hline $\begin{array}{l}\text { Autres (principalement } \\
\text { professions libérales) }\end{array}$ & 2,2 & 0,5 \\
\hline Au 31/12/1984 & \multicolumn{2}{|c|}{$\begin{array}{l}\text { D'après la Revue française des Télécommunications, } \\
n^{\circ} 55, \text { avril } 1985\end{array}$} \\
\hline
\end{tabular}

de données. Certes, ce tableau ne fait pas apparaître explicitement les PME et ne permet pas de mesurer si l'objectif d'ouverture à ce type d'entreprises est rempli, mais il montre la diversité des usagers du réseau Transpac.

Le succès de Transpac dans ses premières années vient certes de la satisfaction d'une demande, mais aussi d'une stratégie commerciale qui s'est avérée efficace. Dès avant son ouverture, Transpac a joui d'une action « précommerciale » par des études de tarifs et l'appui sur un groupement de gros utilisateurs, le GERPAC. Comme le rappelait P. Picard, autre acteur essentiel dans l'histoire du réseau, qui assura notamment la fonction de Directeur Général de Transpac, une première version des tarifs garantis à l'intérieur d'une fourchette de plus ou moins 10\%, est annoncée au SICOB 1976. Cette anticipation commerciale a été bénéfique. De plus la concertation entre techniciens et utilisateurs s'est poursuivie, par le biais notamment de la société UTIPAC, à son origine composée de très gros utilisateurs (18) du secteur nationalisé ou privé, de l'industrie ou de la banque, qui acquièrent une partie du capital de Transpac.

Novateur dans ses choix techniques (commutation de paquets), de tarification, efficace dans son lancement commercial, soutenu par une équipe motivée (des hommes comme P. Picard, G. Pichon, R. Després, Y. Le Bars, G. Simonet, et bien d'autres), répondant à des besoins (contre ce mythe trop fréquent de l'action volontariste d'une DGT suscitant par son offre une demande qui n'aurait pas préexisté), le réseau
Transpac connaît des années 1979 aux années 1985 - I'horizon vers lequel la DGT s'était projetée pour évaluer le succès de son entreprise - un développement important dont témoigne la courbe des accès directs au réseau.

Cependant ce succès commercial, que montre également l'évolution du chiffre d'affaires de la société Transpac, est aussi un succès technique, et la courbe peut être complétée par celle de la capacité installée (page suivante). Transpac a su dépasser chaque année les limites physiques de connexion (sans éviter, comme cité précédemment, quelques difficultés en 1985).

\section{Apports et limites de cette courbe}

La courbe des accès directs permet, comme nous venons de le voir, de mesurer le succès de Transpac au regard des prévisions faites dans la phase initiale de lancement du réseau. Elle reflète la réussite de ce réseau « au service des entreprises » pour reprendre la formule de P. Fortin dans son article de la Revue Française des Télécommunications, ( $n^{\circ} 55, p 34$ et ss). Celui qui s'intéresse à une histoire de la société Transpac pourrait mesurer la bonne santé de l'entreprise dans ces années 1979-1985 en couplant l'analyse des accès directs, du chiffre d'affaires et l'évolution du personnel (ce sont ces trois données que citait G. Pichon). On pourrait y adjoindre une courbe du trafic (par jour et par abonné, le trafic passe par exemple de 3,7 Mbits/sec en 1983 à 4,2 Mbits/s en 1984) et de la capacité installée, mais aussi analyser les délais de raccordements (25 semaines en 
1983, 17 semaines en 1984), ou la qualité du service, afin d'avoir une idée plus précise de l'évolution technique et qualitative du réseau.

Seule, cette courbe ne permet ni d'évaluer la réussite technique du réseau, ni la réussite commerciale de la société. Elle n'informe ni sur la part des PMl et PME parmi les usagers du réseau, ni sur les accès par le réseau téléphonique ou télex, ni sur le « trafic minitel " qui transite via Transpac et qui représente, début 1985, 6\% du trafic total Transpac, et en 1984, 2000 communications vidéotex simultanées sur Transpac en heure chargée. Rappelons que la Cour des Comptes évalue qu'en 1989 le minitel représente 50\% du chiffre d'affaires de Transpac.

Aussi cette courbe des accès directs commerciaux est représentative d'une vision de Transpac comme réseau "au service des entreprises" mais ne mesure pas son succès auprès du grand public ensuite, par le biais du vidéotex. Mais cette vision est celle qui a présidé à la création de Transpac, elle est dans l'esprit des télécommunicants, dans les chiffres de G. Pichon comme dans ceux des auteurs de l'ouvrage Télécommunications diffusé en interne par France Télécom, Iorsqu'ils livrent des données allant de 1986 à 1991 qui continuent de s'appuyer sur les accès directs.

En choisissant d'arrêter cette courbe en 1985, nous avons souhaité montrer les débuts du réseau Transpac et en quoi celui-ci a su apporter, en ce début des années 1980, une réponse efficace aux besoins de transmissions de don-

\section{capacité installée en terme d'accès à Transpac (1979-1985)}

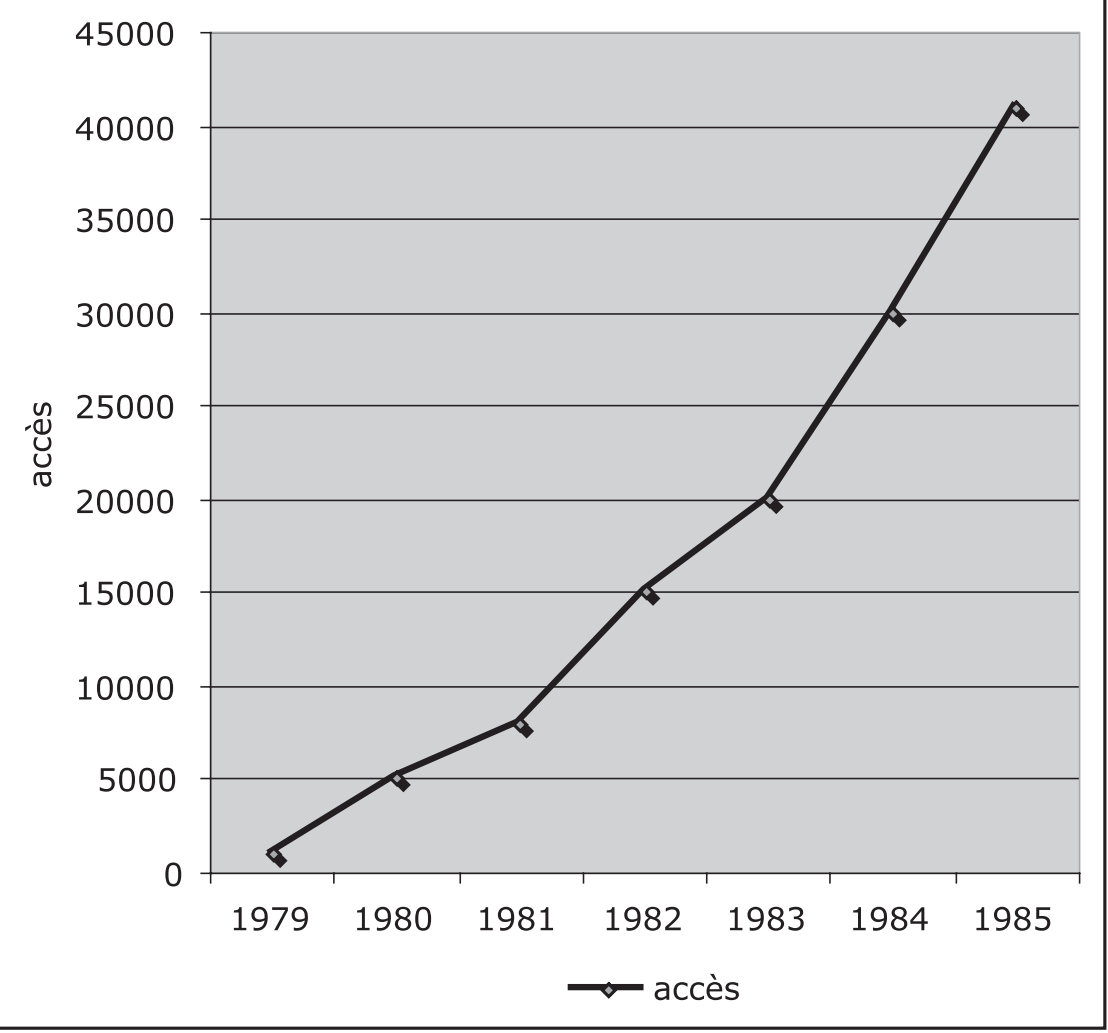

nées. Au-delà, la courbe poursuit sa croissance atteignant en 199188500 accès directs. Transpac a su s'adapter et suivre les évolutions du monde des télécommunications: en 1991 les liaisons utilisées sont des liaisons numériques Transmic, et à cette date le réseau Transpac s'interconnecte au RNIS (Réseau numérique à intégration de services, mis en service en France à la fin de 1987). Mais au début des années 1990, Transpac n'a plus le monopole du service des entreprises, France Télécom a complété son offre en réseaux d'entreprises par Transfix (39000 liaisons en 1991) et Numéris
(150000 canaux B en 1991). La déferlante IP complexifie encore la situation vers 1995.

En aval donc de cette courbe des accès directs commerciaux à Transpac (1979 - 1985), l'évolution du réseau Transpac ouvre d'autres champs d'étude, celui des réseaux numériques, celui de la politique de France Télécom en matière de service aux entreprises, celui de l'arrivée d'IP et des évolutions induites pour Transpac. Aussi, que l'on fasse le choix d'une courbe retraçant les débuts de Transpac ou son évolution ultérieure, il apparaît en tous cas clairement que l'étude de ce réseau est 
Flux $n^{\circ} 62$ Octobre - Décembre 2005

transversale à plusieurs problématiques

essentielles pour l'appréhension de

l'histoire des télécommunications et des

réseaux.

Valérie Schafer

Agrégée d'Histoire-Géographie

Doctorante Université Paris-Sorbonne

(Paris $\mathrm{N})-\mathrm{CRHI}$

\section{Notes}

(1) G. Pichon, « Les débuts du réseau public français de commutation de données par paquets: Transpac ", Actes du $7^{\circ}$ colloque sur l'histoire de l'informatique et des transmissions, 1618 novembre 2004, Éditions IRISA/INRIA-Rennes, 2004, p 45-70.

(2) G. Pichon a été impliqué dès le début dans l'aventure Transpac, puisqu'il entre en Mai 1974 au CCETT (Centre Commun d'Études de Télévision et de Télécommunications) dans l'équipe de Rémi Després, un des principaux ingénieurs à l'origine de Transpac. G. Pichon se consacrera jusqu'en 2000 à Transpac, au cours d'une carrière commencée début 1979 comme directeur adjoint et qui le mène de 1996 à 2000 au poste de conseiller du président de Transpac et directeur de la sécurité.

(3) F. du Castel (dir.), Les Télécommunications, X, A Descours, BergerLevrault International, Paris, 1993, 799 p., p 299.

(4) G. Pichon, " Les débuts du réseau public français de commutation de données par paquets: Transpac ", Actes du $7^{\circ}$ colloque sur l'histoire de l'informatique et des transmissions, 1618 novembre 2004, Éditions IRISA/INRIA-Rennes, 2004, p 45-70.

(5) Idem, p 65.

(6) CAC 19870748, article 53, dossier Télécommunications produits, bilan 1981.

(7) Selon la direction du programme Télétel, le nombre d'appels non aboutis par suite d'encombrements du serveur ou d'erreur d'aiguillage, ou d'occupation du point d'accès, est de 34\% sur l'ensemble des réseaux et 39\% sur le réseau kiosque.

(8) http://www.sutherla.dircon.co.uk /minitel/cour_des_comptes.htm
(9) Les réseaux s'appuyaient sur la commutation de messages, ou de circuits jusqu'aux travaux du CNET et de I'IRIA sur la commutation de paquets, découverte par les Français aux EtatsUnis en 1969 (technique expérimentée par Paul Baran, Licklider ou le réseau Arpa, ancêtre du réseau Internet).

La commutation de circuits consiste à commuter des circuits en aboutant des artères pour constituer pour chaque demandeur « une voie réservée » pour la durée de la transaction. C'est le cas par exemple dans le réseau Caducée, premier réseau spécialisé dans la transmission de données français lancé par le CNET et la DGT en 1971. Mais cette technique n'est intéressante que si les opérations se déroulent en continuité et s'il n'y a pas trop de taux de silence, ce qui représente sinon un gaspillage des ressources allouées. D'où le recours possible à la commutation de messages. Dans ce cas les réseaux sont constitués de circuits spécialisés reliant des centres de commutation entre eux, et de circuits reliant les abonnés aux centres. L'abonné transmet le message à son centre de rattachement qui stocke le message et après avoir trouvé un circuit disponible effectue la transmission, vers le centre destinataire ou un centre intermédiaire. Le message reçu par un centre intermédiaire est vérifié et relayé de centre en centre jusqu'à son centre de destination. Cette technique induit certes un délai de transmission plus long, et qui augmente avec la taille du message, mais en contrepartie, si le délai n'est pas critique, on peut utiliser une plus grande fraction de la capacité des circuits. La commutation de paquets, proche de la commutation de messages, résout ces deux problèmes, de « gaspillage " et de lenteur. Les messages sont découpés en paquets. Ils sont accompagnés d'informations de service qui les identifient pour permettre leur acheminement vers la destination choisie, puis ils sont pris en charge par le réseau de transmission constitué de commutateurs spécialisés chargés de reconnaître la présence d'un paquet, d'examiner les données de service contenues dans chacun, de détecter les erreurs. Un tel système permet une utilisation optimale des lignes de transmission par partage entre les utilisateurs. Une ligne n'est « allouée » à un utilisa- teur que le temps nécessaire à la transmission d'un paquet au nœud suivant. En fonction de l'état du réseau (charge, pannes de ligne ou de nœud), les paquets emprunteront un itinéraire différent, permettant d'atteindre le destinataire dans les meilleures conditions.

(10) Entretien avec Philippe Picard du 1 1er février 2003 (il a été notamment Directeur Général de Transpac après en avoir été le chef de projet).

(11) CEPT, Réseaux publics de données, $2^{\ominus}$ Édition, 1979, 241 p, p. 82-84.

(12) "Quand au début des années 1980 on s'est demandé quel réseau utiliser pour le minitel, (je ne parle pas de l'annuaire électronique, qui ne posait pas ces problèmes de facturation, mais des services télématiques, et en particulier cette invention fantastique qui a été un des deux facteurs de réussite du minitel, à savoir la facturation kiosque), il y avait des débats à l'intérieur de France Télécom, car comme Transpac était une société extérieure, certains disaient qu'ils ne voulaient pas lui confier leurs intérêts, et voulaient construire un réseau spécifique plutôt qu'utiliser Transpac pour véhiculer le trafic minitel. (...) Transpac a été choisi car il était le seul capable pour chaque facturation de savoir qui émettait et surtout qui recevait, donc il était capable de calculer tous les éléments de la facturation: durée, volume, numéro du serveur informatique demandé, etc. ». Entretien avec

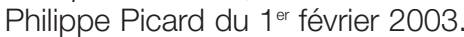

(13) Le Matin, 28 mars 1979.

(14) Le Nouveau Journal, 6 avril 1979.

(15) Ainsi quand est prise en 1973 la décision d'envisager la réalisation d'un réseau public de transmission de données par paquets, la composition du parc des terminaisons est au $1^{\text {er }}$ janvier de 4418 liaisons spécialisées sur un total de 8255 , soit plus de la moitié. (Article des Échos du 7 Mai 1973).

(16) CEPT, Réseaux publics de données, $2^{\ominus}$ Édition, 1979, 241 p., p.82-84.

(17) Simon Nora et Alain Minc, L'Informatisation de la société: rapport à $M$. le Président de la République, Paris, La Documentation française, 1978, 163 p.

(18) En 1979, on compte par exemple parmi les abonnés à Transpac Saint-Gobain-Pont-à-Mousson, UAP ou Renault. 